\title{
Impact of Energy-Efficient and Eco-Friendly Green Computing
}

\author{
Shivangi Sharma \\ Department of Computer \\ Science and Engineering \\ JMIT, Radaur \\ Yamuna Nagar, India
}

\author{
Gaurav Sharma, PhD \\ Department of Computer \\ Science and Engineering \\ JMIT, Radaur \\ Yamuna Nagar, India
}

\begin{abstract}
With the abrupt advancement in technology, every business organization aspires to migrate to cloud. The technology of cloud computing makes use of the huge data centers that present multiple issues such as extensive amounts of energy consumption, dissipation of lots of heat and methane, carbon dioxide, etc. like deadly green-house compounds and gases. In order to solve all the above stated issues of cloud computing, green computing came out as an improvement over the conventional cloud technology with energy-efficiency, security and environmental-friendliness. The two of the Linear power model and the Low Powerblade model are the existing ones, which employ four Virtual Machine scheduling algorithms for the calculation of consumption of power, are not very eco-friendly and energy efficient. These limitations of the two power models call for the requirement of a better and more efficient power model. Therefore, the research work is motivated to devise such a power model that ensures energy-efficient results and proves it environment-friendly when applied on the data centers in a secure way when compared with the two existing power models on the GreenCloud Simulator.
\end{abstract}

\section{General Terms}

Carbon footprints, Heat dissipation, Eco-friendly computing technology, Energy consumption, Resource utilization.

\section{Keywords}

Green Computing, Power models, Scheduling algorithms, GreenCloud Simulator, Energy efficiency, Virtualization, Virtual Machine Migration, Load Balancing, Server Consolidation.

\section{INTRODUCTION}

\subsection{Definition}

Green computing can be defined as a technology in recent trend that has emerged as an improvised version of the cloud computing technology [1] due to the concern for environment and sustenance. Technically, green computing is such a technology for computing that rapidly provisions a convenient access to the shared configurable computing resources [2] dynamically on the demand of its service consumers with least or without intervention of the service provider [3], while preventing any environmental harm and wastage of resources [4].

In short, green computing technology is the one that involves the task of computing along with the responsibility towards environment. The concept behind the technology revolves around the energy efficient servers and peripherals at several cloud data centers, efficient use of resources and management of the 'e-waste' produced [2] [5]. The primary technologies contributing to achieve green computing are Cloud computing, Green Data Center, Virtualization, etc. [6].

Green IT, a similar term, involves all the IT solutions that save energy at numerous levels of use including hardware, software and services [7].

\subsection{Vision}

The green computing aspires to achieve the aim of designing a highly power efficient, secure, environment-friendly and best performance computing system [8]. Green Cloud computing is envisioned to achieve not only efficiency in managing the allocation and working of the several operational resources including infrastructure, but limited consumption of energy too so that the future of the computing technology is sustainable [9]. Simultaneous rise in energy efficiency and fall in the electrical power consumption is the motto of the green computing technology [6].

\subsection{Motivation}

The reason behind the development of green computing lies with the limitations of its parent technology i.e. cloud computing, as the expenses and maintenance of the infrastructure is not the only issue, but other issues include its environment-unfriendliness, enormous energy expenses and the extensive emission of heat and carbon compounds out of the data centers due to the numerous cooling servers installed and are functional inside them [2]. So, the cloud service vendors improvised the cloud technology, ensured that all these drawbacks of cloud are overcome by the newer technology and then, finally named it 'Green Computing' [10].

\subsection{Features of Clouds enabling Green computing}

As green computing has evolved from cloud computing, there are some common features of the former technology that inspires the latter one [4] that are presented as follows:

\subsubsection{Dynamic Provisioning}

In the conventional technology, the IT companies used to deploy far more data centers and infrastructure to fulfil the worst case requirements. Such an arrangement, known as over-provisioning [11] [4], brings a number of added issues like excessive power use, dissipation of heat and harmful carbon compounds leading to extensive financial losses for the organization [12]. Also, to manage peak workloads only for short spans yearly, keeping a number of servers functional for the entire year is not at all an efficient option. In green computing, this problem can be solved by the help of live migration [13], which means the virtual machines in the system are live migrated to some other host if there is a requirement of more resources from the user side. Then, the 
cloud vendor monitors, determines the requirement of the user and finally, allocates the resources accordingly. Such a technique is Load balancing and for all those applications requiring lesser resources than allocated can be server consolidated. So, green computing is dynamic and provisions the resources [14] according to the current and changing requirements of the user [4].

\subsubsection{Multi-tenancy}

Multi-tenancy refers to the approach where the service providers provision the entire infrastructure and other resources to multiple companies [15] and thus, lessen the overall energy usage and the vast amount carbon emissions. Also, multi-tenancy brings out the generalized pattern of energy for different businesses, which drastically improves the efficiency of energy saving [16].

\subsubsection{Server Utilization}

Generally, the traditional cloud infrastructure gives nearly 5 to 10 percent of utilization level when run [17]. After employing virtualization in green technology, several applications can be simultaneously hosted that further leads to improvement up to $70 \%$ in utilization levels and finally, noticeable reduction in the count of active servers and power usage.

\subsubsection{Data center Efficiency}

Another important feature of green computing lies with the power efficiency of data centers and affects the total energy to be used in the system [4].

\subsection{Metrics}

The most important task to perform in order to reduce the total amount of power consumed while the data center is in operation is use some metrics to actually measure it [18]. This is the reason, power models are always constructed that they provide support in determining the power used by various subsystems of the running data centers and also help in knowing the scope of limiting it. Green Grid has put an effort in this direction [32] by proposing two such metrics as follows:

\subsubsection{Power Usage Effectiveness (PUE)}

PUE is a metric that determines the ratio of energy consumed for facility and that spent on overhead [20] using following formula:

$$
\text { PUE }=\frac{\text { Total Power Consumed on the specific facility }}{\text { Total Power Consumed on an IT equipment }}
$$

Ideally, the value of the metric PUE is 1.0 , which denotes $100 \%$ efficiency [35] or the entire electrical power is being used by the IT equipments only [18]

\subsubsection{Data Center Infrastructure Effectiveness (DCiE)}

$\mathrm{DCiE}$ is a metric that determines the ratio of energy spent on overheads and that spent on the specified facility [20] using the following formula.

DCiE $=1 /$ PUE

$=$ Total Power consumed on an IT Equipment $\times 100 \%$ Total Power consumed on the specific facility

For both the above metrics, Total Power consumed on the specific facility is the amount of electrical power measured by the utility meter denoting the power used by the data center [4]. Total Power consumed by IT Equipment is the amount of electrical power consumed in the storage, routing, processing and the entire management of the data within the data center [4].

\subsubsection{Green Power Usage Effectiveness (GPUE)}

GPUE is a metric to overcome the drawbacks of the metric PUE and given by the renowned organization named GreenQloud. Those drawbacks of PUE metric are as follows:

- Completely time and location dependent.

- Involves ignoring certain parameters by the data centers to reduce its rating.

- Applicable only on the dedicated data centers.

- Can support efficient use of power, but can't be used for the comparison of data centers.

The metric GPUE is more practical to use, where $\mathrm{X}$ is a value between $0-3$.

Therefore, GPUE yields values from a wider range and higher resolution in comparison to PUE.

Also, the metric GPUE has already been declared an excellent metric to measure the greenness quotient present while the data centers are running [18].

\subsection{Principles and Strategies}

There are some basic principles [21] and strategies [22] by use of which green computing can be achieved as follows:

\subsubsection{Switch off the facilities when not in use.}

Switching on the facilities only when there is a requirement can reduce the power consumption levels. An example of sleep scheduling can be considered as a power saving technique in WSNs that allows the sensor nodes to be asleep and awake dynamically [23].

\subsubsection{Transmit least data.}

Transmitting data such as multimedia data takes up energy in enormous amounts. So, it is recommended that only the data that is very important to send should be sent. Predictive data delivery is one such technique that works on the basis of user behaviour analysis [21].

\subsubsection{Route the data properly.}

Another principle is regarding the routing of the data when transmitted. Routing schemes employed should be power efficient while choosing the data path and tend to minimize its length [21].

1.6.4 Balance processing with communications. Aggregating and selecting the data from heterogeneous data sources limits the data for transmission that further improves the efficiency in saving energy [21].

\subsubsection{Use power efficient electrical components.}

The use of energy saving devices is encouraged such as liquid crystal display (LCD) monitors and notebook computers [7] in place of cathode ray tube (CRT) monitors and desktop computers [22].

1.6.6 Promote using renewable sources of energy. Renewable energy sources like water, sun, air, timber and biomass etc. that can be replenished and reused that consequently, minimize the discharge of environment unfriendly elements into it [21]. 


\subsubsection{Encourage the use of Nano Data Centers} (NaDa).

A newly developed platform to implement distributed computing named as Nano Data Centers. Several interconnected and small $\mathrm{NaDas}$ spread all over are preferred over those conventional large data centers. By this approach, nearly $25 \%$ of the electrical power can be saved [22].

\subsubsection{Automatically switch off external storage.}

Hard drives can be programmed to automatically get switched off after a prescribed period of inactivity [7].

\subsubsection{Limit the cooling Requirements.}

In order to improvise the electrical energy requirements by continuously sensing the temperature of air of the outside world and if it is critically low, then direct cooling is provided by the refrigerator [22].

\subsubsection{Decrement CPU Power Dissipation.}

Processing units get charged by consuming electrical power and perform the required processing, switching and cooling for multiple devices. Meanwhile, a large amount of energy is dissipated as heat in the environment, which can be reduced using free cooling strategy [22].

\subsection{Methodologies}

Green computing is a standard technology that follows four basic methodologies [24] in order to keep sustainability and computing balanced. These methodologies are presented in the figure 1 below and the table1 following it [7].

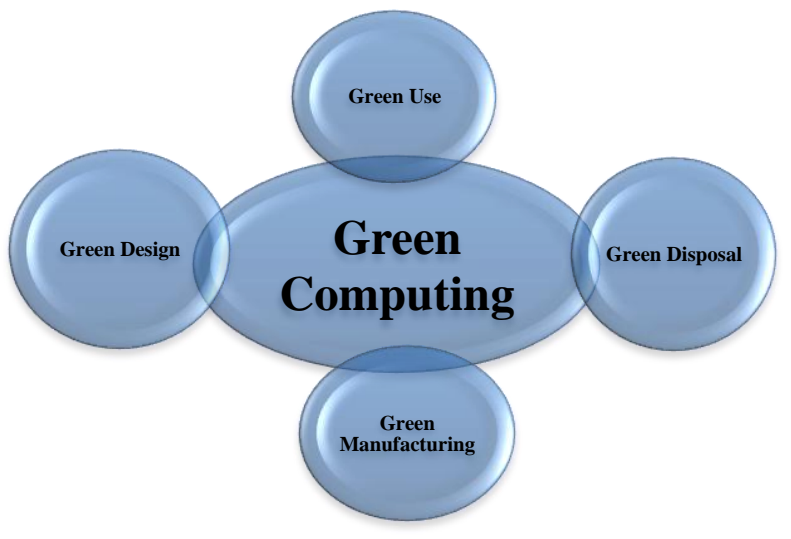

Figure1: Methodologies for Green Computing

Table 1: Uses of methodologies for Green Computing

\begin{tabular}{|c|c|c|}
\hline Sr. No. & $\begin{array}{c}\text { Name of } \\
\text { Methodology }\end{array}$ & Use of Methodology \\
\hline 1. & Green Use & $\begin{array}{c}\text { Limiting the amount of electrical } \\
\text { energy to be consumed by the data } \\
\text { centers and other IT equipments. }\end{array}$ \\
\hline 2. & Green Design & $\begin{array}{c}\text { Designing eco-friendly as well as } \\
\text { energy-saving data centers and all } \\
\text { other hardware components. }\end{array}$ \\
\hline 3. & $\begin{array}{c}\text { Green } \\
\text { Manufacturing }\end{array}$ & $\begin{array}{c}\text { Developing the IT equipments and } \\
\text { peripherals while ensuring there is } \\
\text { no negative impact on the nature. }\end{array}$ \\
\hline 4. & Green Disposal & $\begin{array}{c}\text { Using the old peripherals if they } \\
\text { function properly and recycling } \\
\text { them when they can't be used. }\end{array}$ \\
\hline
\end{tabular}

\subsection{Techniques for Green Computing}

Numerous techniques [3] are used to accomplish the implementation of green computing as follows:

- $\quad$ Green Data Center [20]

- Virtualization [5]

- Carbon Aware Green Cloud Architecture [17]

- Cloud Broker Architecture [25]

- $\quad$ LPT and FPRRT [6]

- Live Migration [13], including Server Consolidation [26] and Load Balancing [27].

\subsection{GreenCloud Simulator}

Simulation can be defined as an approach of emulating all the scenarios of a system, which is not feasible to develop, run and test on the real grounds initially, with the help of software before its actual development and deployment [28]. GreenCloud is the best suitable simulator for simulating a given problem of green computing [29]. So, GreenCloud simulator is being used in the research.

\subsubsection{Steps of Installation of GreenCloud Simulator}

The steps of installation of GreenCloud simulator [28] are presented as follows:

Step 1: Download the software package of GreenCloud simulator and unpack it.

Step 2: Install it successfully by accessing the specified directory and then executing the defined script ./install.sh.

Step 3: Finally, execute the ./run script.

Step 4: Now, start using the simulator as the dashboard is displayed after opening show-dashboard.html file.

\subsubsection{Scripts for Simulation}

The GreenCloud simulates by the help of several important TCL files, or scripts, stored in ./src/scripts/ directory [28]. The file main.tcl decides the two important parameters, the data center topology as well as simulation time, and runs a set of scripts further in the table 2 as follows:

Table2: Scripts used in simulation by GreenCloud.

\begin{tabular}{|c|c|c|}
\hline Sr. No. & $\begin{array}{c}\text { Simulation } \\
\text { Scripts }\end{array}$ & Task Performed \\
\hline 1. & $\begin{array}{c}\text { setup_params.t } \\
\text { cl }\end{array}$ & $\begin{array}{c}\text { Defines the customizable general } \\
\text { configuration of the servers, } \\
\text { switches, tasks, etc. }\end{array}$ \\
\hline 2. & topology.tcl & $\begin{array}{c}\text { Selects and implements the data } \\
\text { center network topology. }\end{array}$ \\
\hline 3. & dc.tcl & $\begin{array}{c}\text { Creates the data center servers and } \\
\text { VMs dynamically. }\end{array}$ \\
\hline 4. & record.tcl & $\begin{array}{c}\text { Records the results after execution of } \\
\text { procedures. }\end{array}$ \\
\hline 5. & user.tcl & $\begin{array}{c}\text { Defines the number and behaviour of } \\
\text { cloud users. }\end{array}$ \\
\hline 6. & finish.tcl & $\begin{array}{c}\text { Determines the output and maintain } \\
\text { simulation statistics. }\end{array}$ \\
\hline
\end{tabular}




\subsubsection{Features of GreenCloud Simulator}

GreenCloud simulator comprise of features as follows:

- Developed as an extension of the popular network simulator Ns2 [29].

- Capable of compiling two important languages, $\mathrm{C}++$ and Tool Command Language (TCL). The backend is programmed in $\mathrm{C}++$ and the TCL is used for developing the interface and the visualization working environment [29].

- The simulation environment utilizes two models for calculating the power consumption that are Linear Power Model and Low Power Blade Model [28].

- Round Robin scheduler using host and using VMs and Green scheduler and Green scheduler using VMs are the four scheduling algorithms that are used according to different requirements by these power models [30].

- Works on the basis of the 3-Tier Data center Network Architecture [29] as shown below in figure 2, which uses L3 and L2 switches in its layers. A single switch is employed within the core network layer, two within the aggregation layer and 144 PMs, arranged in TOR topology, within the access layer [30].

- Selection of the power model, count of servers and switches and users are all customizable and can be changed dynamically using the simulator [28].

- Compatible to work with Linux platform. Any linux flavor can be used. Ubuntu 12.04 versions is been used in the research [28].

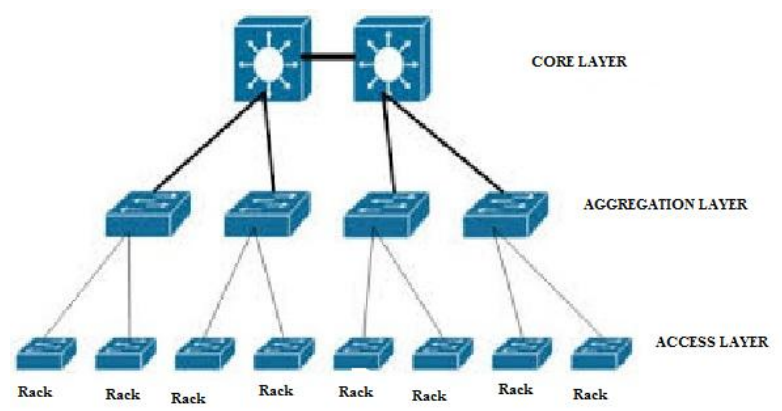

Figure 2: The 3-Tier Data center Network Architecture

\subsubsection{Power Models}

A power model is a prototype or a virtual implementation of scheduling algorithms on data centers to determine the power consumption by them using the specified algorithms.

\subsubsection{Types of Power Models in GreenCloud}

Till now, the GreenCloud simulator uses its two in-built power models [28] as follows:

\section{a) Linear power model}

The model supports the following features:

- Racks with mounted servers are used.

- Power chord and network cable are pre-requisites for its functioning.

- Ideally, the rack configuration is nearly 19 inches wide and 1.75 inches tall

- 42 discrete computer devices can be accommodated in a single rack.

b) Low Power Blade Model

The model supports the following features:
- Several components are removed in order to save electrical power and storage space.

- The blade enclosure may hold multiple blade servers that provide services like power, cooling, networking etc.

- 1440 servers per rack can be accommodated.

\subsubsection{VM Scheduling in Green Computing}

Scheduling is a vital section in green computing whose goal is to ensure the maximization of the use of the resources by its tasks while minimizing its processing time. VM scheduling is the one that schedules and maps the VM requests with the physical machines or servers (PMs) in the data center [30].

\subsubsection{Working of VM Scheduling Algorithms}

A VM scheduling algorithm is a stepwise procedure, which optimizes energy, expenses, time period and security, is bifurcated in three levels of working:

Level 1: A suitable PM is determined for multiple VMs.

Level 2: An appropriate scheme for provisioning is selected to apply on the VMs.

Level 3: Finally, the scheduling is applied on the VMs.

\subsubsection{Categorization of VM Scheduling Algorithms}

There are several categories of VM Scheduling algorithms [30] presented as follows:

\section{a) Random VM Scheduling Algorithm}

In this algorithm, the assignment of a given task to a VM available instantaneously is done randomly [28]. Such a scheduling algorithm has a merit that it is not at all complex, but at the same time suffers from the demerit of long waiting time for a smaller task before it is served, if a longer task is selected to be served randomly [30].

\section{b) Round Robin VM Scheduling Algorithm}

In this algorithm, the assignment of the all the tasks to the VMs available instantaneously is completed in a round-robin fashion and each task is given a slice of the total time to get served [28]. The merit of the algorithm lies in its fair distribution of tasks among all the available servers, achieving load balancing and avoiding delay and congestion [30].

\section{c) Green VM Scheduling Algorithm}

In this algorithm, the scheduling of the tasks is carried out by an energy-efficient scheduler [28] known as Green Scheduler. The scheduler keeps track of the buffer capacity of network switches, prevents congested routes, set the idle servers to sleep mode and takes care of the less-loaded servers [30].

\section{LITERATURE SURVEY}

The research work performed in this field by different researchers is presented as follows:

Vinay P. Viradia et al. [1] described the benefits and challenging issues of the cloud concept that motivate the adoption of a newly developed and an improved version of cloud technology i.e. green cloud computing.

Jayant Baliga et al. [2] analyzed the approach of energy consumption in switching, transmission, data storage and data processing while implementing both public as well as private clouds.

Shivangi Sharma et al. [6] conducted a comparative analysis after analyzing all the approaches and techniques for implementing efficient use of energy and virtualization in the 
green technology. Thus, the use of green computing for cloud computing is encouraged in the paper.

Shalabh Agarwal et al. [7] explored the concept of green computing from the viewpoint of business and IT, implemented Green IT in order to ensure cost savings in an eco-friendly manner and also identified several approaches of implementing green computing in ICT sector.

Wanneng Shu et al. [8] presented an improvised algorithm known as clonal selection on the basis of duration, expenses and the model for calculating the energy consumed in the cloud environment using CloudSim. Also, the parameters like amount of energy consumed and make span in association with the resources to be provisioned are considered.

Si-Yuan Jing et al. [10] explained all the existing research work on the efficient use of power and its saving in the IaaS model of the cloud computing system that eats up superabundant quantity of energy in a cloud computing system using green cloud computing.

Jorge Werner et al. [11] introduced a model that ensures the target characteristics of services in Green Computing such as dynamic scalable, Quality-of-Service standard and diminished energy utilization and solves the current problem of over provisioning in data centers w.r.t. the requirement of mapping with the peak load, using CloudSim.

Jaspreet Kaur et al. [13] implemented live migration on the data centers using the two essential performance criteria namely, the total migration duration and the downtime.

Kim Khoa Nguyen et al. [16] presented work in the field of virtual slicing technique, which encourages the saving of electrical energy in enormous amounts and optimal use of the renewable sources of energy. Keeping in view the several server consolidation strategies, an optimal solution strategy is formulated for the problem of assignment of virtual slices and simulated on GSN.

Mueen Uddin et al. [20] proposed an energy-saver and sustainable green ICT model that employs several subset technologies such as cloud, virtualization and eco-friendly parameters in five different phases to perform outstandingly by precisely separating the components of the data center into resource pools distinctly.

Chunsheng Zhu et al. [21] proposed a number of technologies and issues in relation with Green Internet of Things (GIoT).

Amlan Deep Borah et al. [22] encouraged the use of green computing technology to resolve the energy crisis issue and proposed an improvised algorithm design and techniques to fulfil the required energy efficiency. Also, the virtual machine migration has emerged as an energy-aware approach to be implemented on the data centers is included in the paper.

Rohit Narang et al. [28] focused on the issue of efficient energy utilization in cloud technology and implemented the Linear as well as the Low Powerblade power models using different scenarios and scheduling algorithms. The findings show distinguished values for consumed and saved energy, tasks rejected by data center and tasks failed on servers.

Lena Mashayekhy et al. [31] proposed an algorithm that determines the winner by selecting the candidate users, provisioning multiple virtual machines (VMs) with physical machines (PMs), allocating them to the selected candidate users and there exists a payment function for determining the amount that each selected candidate user is required to pay to the specified cloud service provider. The proposed algorithm is robust against the unauthorized users trying to operate the system by editing the allocations of the other users and strategy-proof too.

Tharam Dillon et al. [32] brought to light all the challenges and issues faced by Cloud computing.

Anton Beloglazov et al. [36] proposed an energy conserving and efficient resource allocation and management system for these data centers, by the consolidation of virtual machines continuously in accordance with the existing use of the resources and the network topologies employed.

\section{PROPOSED WORK}

\subsection{Problem Formulation}

The cloud technology, which has become very popular in last few years, suffers major setbacks in terms of efficient use of electrical power resources, dissipation of enormous amounts of heat and extensive discharge of deadly gases and compounds while the data centers are running. Green Computing has come up as a promising solution strategy for all these stated concerns of Cloud technology.

In the GreenCloud simulator that has been used for simulation in the research work, there already exist two power models namely the Linear Power Model and the Low Powerblade Power Model. The performance of these power models can be improved on. Thus, a better and more energy efficient power model needs to be developed that is capable of scheduling and running the data centers in a better way, can save more energy and does not compromise its eco-friendliness for performance.

\subsection{Proposed Work}

In the paper, a new and an improvised power model has been developed that employs different algorithms for scheduling and running the data centers more efficiently. The proposed power model outperforms both the existing power models in terms of energy efficiency as well as sustenance.

\section{RESULTS AND ANALYSIS}

'GreenCloud' is the simulator used for simulation of the proposed power model and comparing its performance with the preceding two power models. For all the results, LM stands for Linear Power Model, LPM for Low Powerblade Power Model, PM* for Proposed Model, PMLM* for Proposed Model using Linear Model and PMLPM* for Proposed Model using Low Powerblade Power Model. In addition to this, the scheduling algorithms are also abbreviated in the simulation results, such as GSVMs for Green Scheduler using Virtual Machines, GS for Green Scheduler, RRVMs for Round Robin using Virtual Machines and RRHs for Round Robin using Hosts. The results are as under:

\subsection{Total Energy}

Total energy represents the amount of electrical energy or power consumed by the IT equipments in the entire system including servers, switches, etc. It is measured in $\mathrm{W}^{*} \mathrm{k}$. By formula,

Total Energy =Server Energy + Network Switches Energy Figure 3 showing total energy consumed by the proposed power model is as follows: 


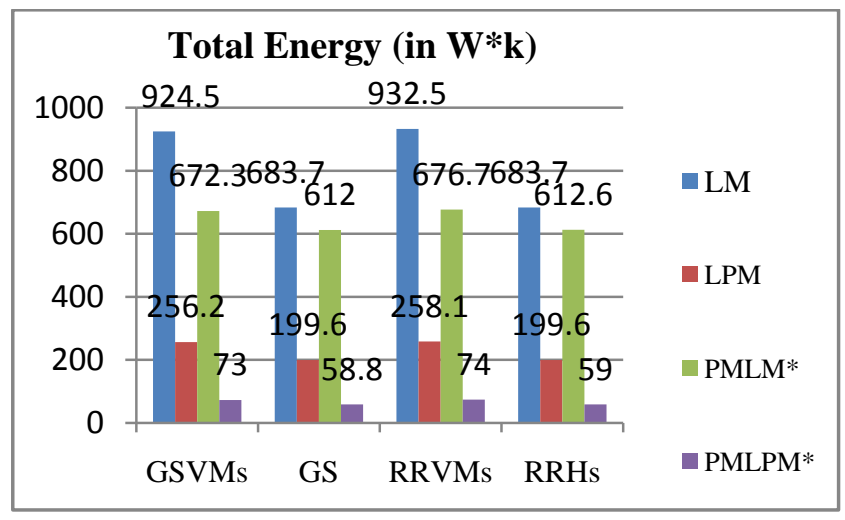

Figure 3: Comparison of Total Energy consumed by proposed power model $(*)$ with the existing models

\subsection{Network Switches Energy}

Network switches energy is the proportionate amount of the total electrical energy or power taken up by the switches only for the proper functioning. Its unit of measurement is $\mathrm{W}^{*} \mathrm{k}$.

By formula,

Switches Energy = Core Energy + Aggregation Energy + Access Energy

In the proposed model, Switches Energy is reduced to $0 \mathrm{~W}^{*} \mathrm{k}$.

Figure 4 showing comparison of switches energy used by the proposed power model with the existing models is as follows:

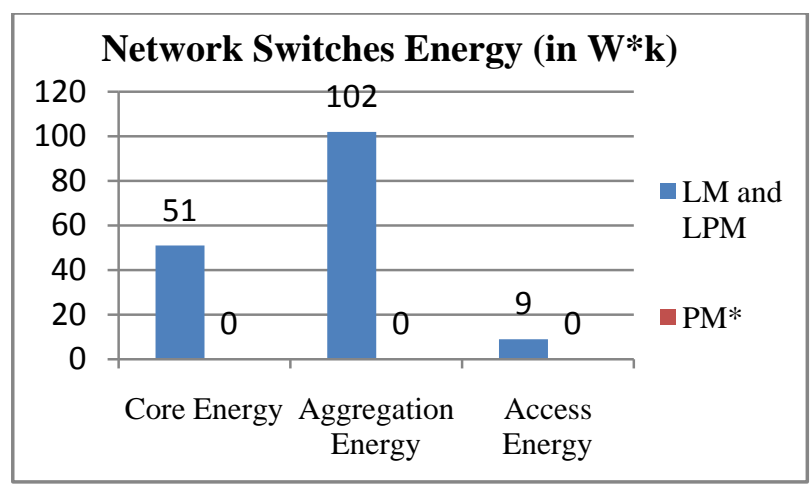

Figure 4: Comparison of Network Switches Energy in Proposed Power Model (*) with the existing models

\subsection{Server Energy}

Server energy is the proportionate amount of the total electrical energy or power taken up by the servers only for the proper functioning. Its unit of measurement is $\mathrm{W}^{*} \mathrm{k}$.

By formula,

Server Energy $=$ Total Energy - Switches Energy, where

Switches Energy $=0$

Thus, Server Energy = Total Energy

Figure 5 showing comparison of server energy used by the proposed power model with the existing models is as follows:

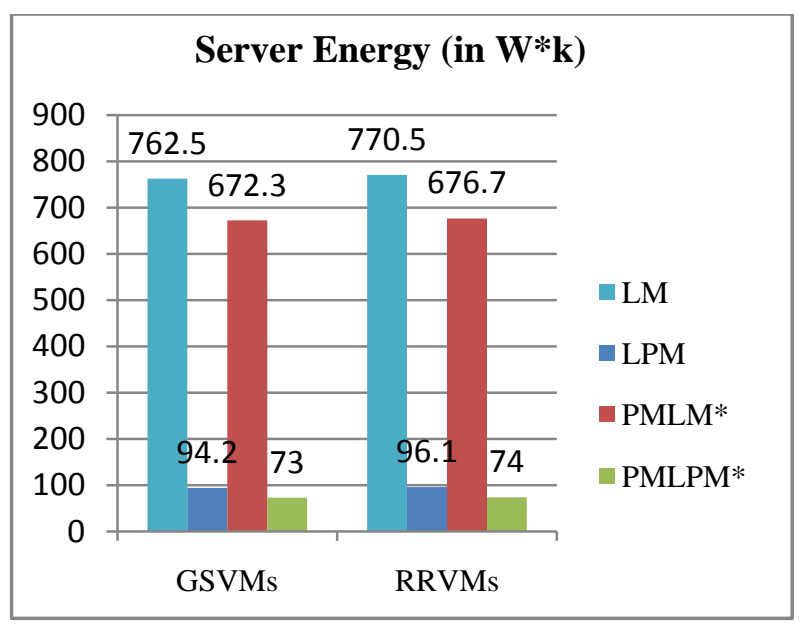

Figure 5: Comparison of Server Energy consumed by proposed power model $(*)$ with the existing models

\subsection{Average Load/Server}

Average Load/Server denotes the total no. of tasks assigned to each server (known as load) divided by the no. of servers. It is measured on a scale ranging from 0 to 1 . Figure 6 showing the comparison of average load per server used by the proposed power model with the existing models is as follows:

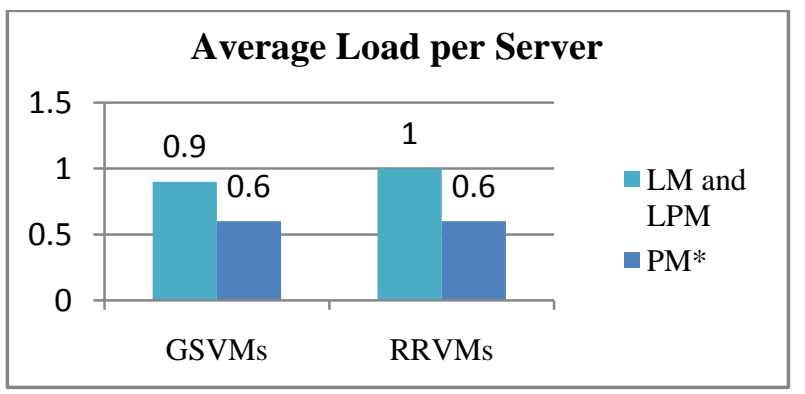

Figure 6: Comparison of Average Load/ server in Proposed Power Model (*) with the existing models

\subsection{Data Center Load}

Data Center Load is the factor denoting the no. of requests executing per DC, or how much it is busy or idle. It is measured as percent (out of 100). The figure 7 shows the comparison of parameter by the proposed power model with the existing models as follows:

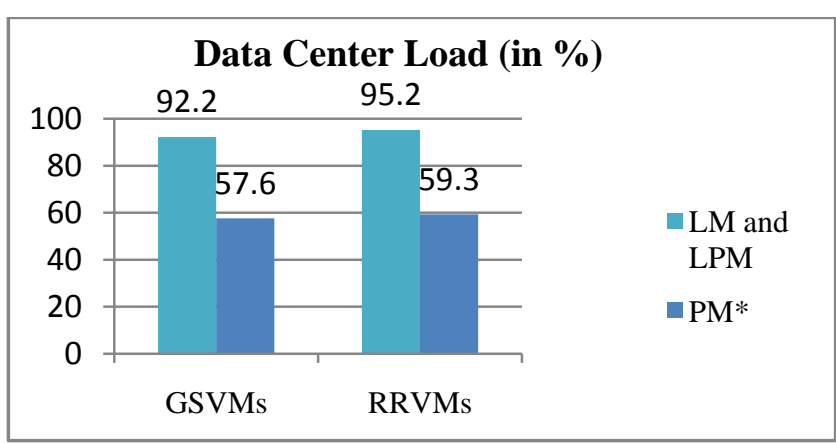

Figure 7: Comparison of Data Center Load in Proposed Power Model (*) with the existing models

Comparative Analysis:

The comparison of the total, server and switches energy consumed by the proposed model with existing models, which prove it energy-efficient and environment-friendly, can be shown with the help of tables 3 , 4and 5 below: 
Table 3: Comparison of Total Energy consumption (in $\left.\mathrm{W}^{*} \mathrm{k}\right)$ by the Proposed Power Model with all the power models

\begin{tabular}{|c|c|c|c|c|c|}
\hline \multicolumn{2}{|c|}{$\begin{array}{c}\text { Power Model/ } \\
\text { Scheduling } \\
\text { Algorithm }\end{array}$} & LM & LPM & $\begin{array}{c}\text { PMLM } \\
*\end{array}$ & $\begin{array}{c}\text { PMLPM } \\
*\end{array}$ \\
\hline 1. & GSVMs & 924.5 & 256.2 & 672.3 & 73 \\
\hline 2. & GS & 683.7 & 199.6 & 612 & 58.8 \\
\hline 3. & RRVMs & 932.5 & 258.1 & 676.7 & 74 \\
\hline 4. & RRHs & 683.7 & 199.6 & 612.6 & 59 \\
\hline
\end{tabular}

'*' denotes the model is a proposed one and does not exist previously.

Table 4: Comparison of Server Energy consumption (in $W^{*} k$ ) by the Proposed Power Model with all the power models

\begin{tabular}{|c|c|c|c|c|c|}
\hline \multicolumn{2}{|c|}{$\begin{array}{c}\text { Power Model/ } \\
\text { Scheduling } \\
\text { Algorithm }\end{array}$} & LM & LPM & $\begin{array}{c}\text { PMLM } \\
*\end{array}$ & $\begin{array}{c}\text { PMLPM } \\
*\end{array}$ \\
\hline 1. & GSVMs & 762.5 & 94.2 & 672.3 & 73 \\
\hline 2. & RRVMs & 770.5 & 96.1 & 676.7 & 74 \\
\hline
\end{tabular}

Table 5: Comparison of Switches Energy consumption (in $W^{*} k$ ) by the Proposed Power Model with all the power models

\begin{tabular}{|c|c|c|c|}
\hline \multicolumn{2}{|c|}{$\begin{array}{c}\text { Power Model/ } \\
\text { Switches Energy }\end{array}$} & LM and LPM & PM* \\
\hline 1. & Core Energy & 51 & 0 \\
\hline 2. & Aggregation Energy & 102 & 0 \\
\hline 3. & Access Energy & 9 & 0 \\
\hline
\end{tabular}

After the comparative results, several points about the proposed model to be discussed are as follows:

- The consumption of the total energy and the server energy is much lesser than the other two models.

- Energy to be used by the network switches i.e. on the core, aggregation and access layers of the 3-tier architecture is reduced to zero.

- Total energy is equivalent to Server energy as switches energy gets reduced to zero.

- The decremented data center load also proves its performance better than the other two.

- The no. of rejected and failed tasks also reduced.

- Server consolidation and load balancing techniques of Virtual Machine Migration are better employed as that of the other two models.

\section{CONCLUSION AND FUTURE SCOPE}

In today's scenario, the users are growing and with that, the overall computing requirements are increasing manifold. The nature is getting short of the valuable sources of energy too. All these facts relate, collaborate and call for the development and employment of energy-efficient systems that provide support to reduce the consumption of energy by the huge and extensively used data centers in the cloud technology. In addition to this, these data centers emit superfluous amounts of heat, deadly gases and harmful carbon compounds. Hence, the green computing has been proven a better technology after solving all the issues of the traditional cloud technology. The GreenCloud Simulator, used for simulation, provides two built-in power models that do not focus much on the factor of energy conservation. Thus, a new power model has been devised, as the research work, which is capable of improving the energy saving parameters such as total energy, server energy, etc. and outperforms both the existing power models. Further, the work can be enhanced by applying task migration in the model to limit both the rejected and failed tasks.

\section{ACKNOWLEDGMENTS}

Genuinely, thanks to all those researchers who published articles and helped others to comprehend the subject of green computing, who contributed in the development of a supreme simulator GreenCloud and finally, to those who developed a presentable and interactive template.

\section{REFERENCES}

[1] Vinay P. Viradia, Chintan M. Bhatt and Hardik S. Jayswal, 'Green Cloud: Implementation and Adoption of Green Data Center', Proceedings of the Second International Conference on "Emerging Research in Computing, Information, Communication and Applications" ERCICA 2014, Elsevier, July 2014, pp. 163-167, DOI: 10.13140/2.1.1043.2962

[2] Jayant Baliga, Robert W. A. Ayre, Kerry Hinton, and Rodney S. Tucker, 'Green Cloud Computing: Balancing Energy in Processing, Storage, and Transport' , Proceedings of the IEEE, vol.1, January 2011, pp. 149167, DOI: $10.1109 /$ JPROC.2010.2060451

[3] Anup R. Nimje, V. T. Gaikwad and H. N. Datir, "Green Cloud Computing: A Virtualized Security Framework for Green Cloud Computing", International Journal of Advanced Research in Computer Science and Software Engineering, vol. 3, Issue 4, pp. 642-646, April 2013, http://www.ijarcsse.com/docs/papers/Volume_3/4_April 2013/V3I4-0125.pdf

[4] Saurabh Kumar Garg and Rajkumar Buyya, 'Green Cloud computing and Environmental Sustainability', Cloud computing and Distributed Systems (CLOUDS) Laboratory, 2011, www.cloudbus.org/papers/CloudEnvSustainability2011.pdf

[5] Chao-Tung Yang, Kuan-Chieh Wang, Hsiang-Yao Cheng, Cheng-Ta Kuo and Ching-Hsien Hsu," Implementation of a Green Power Management Algorithm for Virtual Machines on Cloud Computing", Springer-Verlag Berlin Heidelberg 2011, UIC 2011, LNCS 6905, pp. 280-294, 2011, http://link.springer.com/chapter/10.1007/978-3-64223641-9_24

[6] Shivangi Sharma and Gaurav Sharma, 'A Review on Secure and Energy Efficient Approaches for Green Computing', International Journal of Computer Applications, Volume 138, No.11, March 2016, pp. 2532, DOI: 10.5120/ijca2016909044

[7] Shalabh Agarwal and Asoke Nath, “"Green Computing a new Horizon of Energy Efficiency and Electronic waste minimization": a Global Perspective', 2011 International Conference on Communication Systems 
and Network Technologies, July 2011, pp. 688-693, DOI: $10.1109 /$ CSNT.2011.148

[8] Wanneng Shu, Wei Wang and Yunji Wang, 'A novel energy-efficient resource allocation algorithm based on immune clonal optimization for green cloud computing', EURASIP Journal on Wireless Communications and Networking 2014, SpringerOpen, 23 April 2014, doi:10.1186/1687-1499-2014-64

[9] Wei-Tek Tsai, Xin Sun and Janaka Balasooriya, "Service-Oriented Cloud Computing Architecture", ITNG, 2010, Seventh International Conference on Information Technology: New Generations (ITNG 2010), 2010, pp. 684-689, http://doi.ieeecomputersociety.org/10.1109/ITNG.2010.2 14

[10] Si-Yuan Jing, Shahzad Ali, Kun She and Yi Zhong, 'State-of-the-art research study for green cloud computing', Springer Science+Business Media, LLC 2011, Dec 8, 2011, DOI 10.1007/s11227-011-0722-1

[11] Guilherme Arthur Geronimo, Jorge Werner, Rafael Weingartner, Carlos Becker Westphall, and Carla Merkle Westphall, 'Provisioning, Resource Allocation, and DVFS in Green Clouds', International Journal on Advances in Networks and Services, vol. 7 no 1 \& 2, July 2014, pp. 108-117, DOI: 10.1109/LANOMS.2011.6102263

[12] Yuxiang Shi, Xiaohong Jiang and Kejiang Ye, "An Energy-Efficient Scheme for Cloud Resource Provisioning Based on CloudSim", in proceedings of the IEEE International Conference on Cluster Computing, pp.595-599,2011,

http://ieeexplore.ieee.org/xpl/articleDetails.jsp?arnumber $=6061156$

[13] Jaspreet Kaur, Manpreet Kaur and Sahil Vashist, 'Virtual Machine Migration in Cloud Datacenters', International Journal of Advanced Research in Computer Science and Software Engineering 4(8), August - 2014, pp. 190-193, http://www.ijarcsse.com/docs/papers/Volume_4/8_Augu st2014/V4I8-0331.pdf

[14] Arindam Banerjee, Prateek Agrawal and N. Ch. S. N. Iyengar", Energy Efficiency Model for Cloud Computing," International Journal of Energy, Information and Communications, vol. 3, Issue 6, pp.2942,2013 ,

http://www.sersc.org/journals/IJEIC/vol4_Is6/4.pdf

[15] S.C. Rachana, H.S. Guruprasad," Emerging Security Issues and Challenges in Cloud Computing", International Journal of Engineering Science and Innovative Technology, vol. 3, Issue 2, March 2014, www.ijesit.com/Volume3/Issue2/IJESIT201402_62.pdf

[16] Kim Khoa Nguyen and Mohamed Cheriet, 'Environment-aware Virtual Slice Provisioning in Green Cloud Environment', Article in IEEE Transactions on Services Computing, May 2015, pp. 1-14, http://dx.doi.org/10.1109/TSC.2014.2362544

[17] Saurabh Kumar Garg, Chee Shin Yeo and Rajkumar Buyya, "Green Cloud Framework For Improving Carbon Efficiency of Clouds", January 2011, http://link.springer.com/chapter/10.1007/978-3-64223400-2_45?no-access=true
[18] Chiranjeeb Roy Chowdhury, Arindam Chatterjee, Alap Sardar, Shalabh Agarwal and Asoke Nath, 'A Comprehensive study on Cloud Green Computing: To Reduce Carbon Footprints Using Clouds', International Journal of Advanced Computer Research, Mar 2013, pp. $1-9$

[19] Mueen Uddin, Muhammad Talha, Azizah Abdul Rahman, Asadullah Shah, Jameel Ahmed Khader and Jamshed Memon", Green Information Technology (IT) framework for energy efficient data centers using virtualization," International Journal of Physical Sciences (IJPS), vol. 7(13), pp. 2052 - 2065, 23 March 2012,

http://www.academicjournals.org/article/article1380553649_ Uddin\%20et\%20al.pdf

[20] Mueen Uddin and Azizah Abdul Rahman, 'Energy efficiency and low carbon enabler green IT framework for data centers considering green metrics', Renewable and Sustainable Energy Reviews 16 (2012), Elsevier, pp. 4078-4094, doi:10.1016/j.rser.2012.03.014

[21] Chunsheng Zhu, Victor C. M. Leung, Lei Shu and Edith C.-H. Ngai, 'Green Internet of Things for Smart World' , Article in IEEE Access, vol.3, 2015, pp. 2151- 2162, DOI: 10.1109/ACCESS.2015.2497312

[22] Amlan Deep Borah, Deboraj Muchahary, Sandeep Kumar Singh and Janmoni Borah, 'Power Saving Strategies in Green Cloud Computing Systems', International Journal of Grid Distribution Computing Vol.8, No.1 (2015), Feb 2015, pp.299-306, DOI: 10.14257/ijgdc.2015.8.1.28

[23] Robert R. Harmon and Nora Auseklis, 'Sustainable IT Services: Assessing the Impact of Green Computing Practices', PICMET 2009 Proceedings, Portland, Oregon USA, August 2-6, 2009, pp.1707-1717

[24] Pushtikant Malviya and Shailendra Singh," A Study about Green Computing", International Journal of Advanced Research in Computer Science and Software Engineering, vol. 3, Issue 6, pp. 790-794, June 2013, http://www.ijarcsse.com/docs/papers/Volume_3/6_June2 013/V3I6-0320.pdf

[25] Rajkumar Buyya, Anton Beloglazov and Jemal Abawajy, 'Energy-Efficient Management of Data Center Resources for Cloud Computing: A Vision, Architectural Elements, and Open Challenges', June 2010, http://www.cloudbus.org/papers/GreenCloud2010.pdf

[26] Mingfu Li, Jingping Bi and Zhongcheng Li, 'Improving consolidation of virtual machine based on virtual switching overhead estimation', Journal of Network and Computer Applications 59 , Elsevier, July 22, 2015, http://dx.doi.org/10.1016/j.jnca.2015.07.008

[27] Akhil Goyal and Navdeep S. Chahal, 'A Proposed Approach for Efficient Energy Utilization in Cloud Data Center', International Journal of Computer Applications, Volume 115 - No. 11, April 2015, pp.24-27, DOI: $10.5120 / 20197-2439$

[28] Rohit Narang, Kamal Kumar Sharma and Sharad Chauhan, 'An Energy Efficient Model For Green Computing', International Journal for Research in Applied Science \& Engineering Technology (IJRASET), Volume 3 Issue XI, November 2015, pp. 490-498, http://www.ijraset.com/archive-detail.php?AID=35 
[29] Dzmitry Kliazovich, Pascal Bouvry and Samee Ullah Khan", GreenCloud: a packet-level simulator of energyaware cloud computing data centers," Springer Science+Business Media, LLC 2010, DOI 10.1007/s11227-010-0504-1

[30] Saleh Atiewi, Salman Yussof and Mohd Ezanee, 'A Comparative Analysis of Task Scheduling Algorithms of Virtual Machines in Cloud Environment', Journal of Computer Sciences 2015, 11 (6), pp. 804-812, DOI: 10.3844/jcssp.2015.804.812

[31] Lena Mashayekhy, Mahyar Movahed Nejad and Daniel Grosu, 'Physical Machine Resource Management in Clouds: A Mechanism Design Approach', Article in IEEE Transactions on cloud computing, July 2015, pp.114, DOI: 10.1109/TCC.2014.2369419

[32] Tharam Dillon, Chen Wu and Elizabeth Chang, 'Cloud Computing: Issues and Challenges', 2010 24th IEEE International Conference on Advanced Information Networking and Applications, pp.27-33, DOI 10.1109/AINA.2010.187

[33] Md. Faizul Bari, Raouf Boutaba, Rafael Esteves, Lisandro Zambenedetti Granville, Maxim Podlesny, Md Golam Rabbani, Qi Zhang and Mohamed Faten Zhani, 'Data Center Network Virtualization: A Survey', IEEE
Communications Surveys \& Tutorials, Vol. 15, No. 2, Second Quarter 2013, pp. 909-928, DOI: 10.1109/SURV.2012.090512.00043

[34] Anuj Prasher and Rajkumari Bhatia," A Review On Energy Efficient Cloud Computing Algorithms", International Journal of Application or Innovation in Engineering \& Management (IJAIEM), vol. 3, Issue 4, April 2014 http://www.ijaiem.org/volume3issue4/IJAIEM-2014-0430-107.pdf

[35] Doshi Chintan Ketankumar, Gaurav Verma and K. Chandrasekaran, 'A Green Mechanism Design Approach to Automate Resource Procurement in Cloud', Eleventh International Multi-Conference on Information Processing-2015 (IMCIP-2015), ScienceDirect, Procedia Computer Science 54 (2015), Dec 2015 , pp. 108 - 117, DOI: 10.1016/j.procs.2015.06.013

[36] Anton Beloglazov and Rajkumar Buyya, 'Energy Efficient Resource Management in Virtualized Cloud Data Centers', 2010 10th IEEE/ACM International Conference on Cluster, Cloud and Grid Computing, 2010, pp. 826-831, DOI 10.1109/CCGRID.2010.46 